J. Nonlinear Var. Anal. 5 (2021), No. 2, pp. 319-329

Available online at http://jnva.biemdas.com

https://doi.org/10.23952/jnva.5.2021.2.09

\title{
ON GENERALIZED COMMON QUASI-EIGENVECTOR PROBLEMS
}

\author{
A. FARAJZADEH ${ }^{1}$, A. HOSEINPOUR ${ }^{2,3}$, C.-F. WEN $^{4,5, *}$ \\ ${ }^{1}$ Department of Mathematics, Razi University, Kermanshah, 67149, Iran \\ ${ }^{2}$ Research Center for Interneural Computing, China Medical University Hospital \\ China Medical University, Taichung, Taiwan \\ ${ }^{3}$ Department of Applied Mathematics, National Sun Yat-sen University, Kaohsiug, Taiwan \\ ${ }^{4}$ Center for Fundamental Science, Research Center for Nonlinear Analysis and Optimization, \\ Kaohsiung Medical University, Kaohsiung, 80708, Taiwan \\ ${ }^{5}$ Department of Medical Research, Kaohsiung Medical University Hospital, Kaohsiung, 80708, Taiwan.
}

\begin{abstract}
In this paper, four types of generalized quasi-eigenvector problems in the setting of normed linear spaces are introduced. Some existence results on the solutions of the generalized common quasi-eigenvector problems are established based on the generalized KKM theory.
\end{abstract}

Keywords. Eigenvector; Common quasi-eigenvector problems; Generalized KKM principle; Normed spaces.

\section{INTRODUCTION}

In 1984, Shemsh [1] gave some conditions in which two square matrices $A$ and $B$ have a common eigenvector. There are many results based on Shemsh's results and a number of algorithms in various applied sciences are drived by Shemsh's results; see, e.g., [2]. However, they basically remain formal results which are difficult to use in the presence of round-off errors because the Shemsh's formula is numerically unstable.

In 2001, Li [3] first proved some eigenvector theorems by applying the famous Fan-KKM theorem [4] in infinite-dimensional case. Subsequently, Li and Park [5] obtained some new eigenvector theorems from their equilibrium theorems. Afterwards, Lin and Du [6] studied four types of common eigenvector problems for a family of mappings whose domains are subsets of normed spaces (they called these problems common quasi-eigenvector problems). They established some existence theorems in complex normed linear spaces. Recently, Abreu, MoralesRodrigo and Suarez [7] considered a nonlinear problem and proved the existence of the principal eigenvalues. They also showed the existence and uniqueness of positive solution of their problem arising from population dynamics. The authors in [8] presented an optimization scheme to solve generalized eigenvalue problems (GEP) involving a nonsmooth regularizer. They applied an alternative formulation of GEP where the feasibility set of the model involved the Stiefel manifold. Finally, they showed how the investigated problem improved statistical analysis of

\footnotetext{
${ }^{*}$ Corresponding author.

E-mail addresses: farajzadehali@gmail.com (A. Farajzadeh), a_hosseinpour@outlook.com (A. Hoseinpour), cfwen@kmu.edu.tw (C.-F. Wen).

Received October 11, 2020; Accepted January 7, 2021.
}

(C)2021 Journal of Nonlinear and Variational Analysis 
brain imaging data where the regularizer is derived from other 'views' of the disease pathology, involving clinical measurements and other image-derived representations. Song and Qi [9] applied the Edelstein contraction theorem to present the nonlinear version of the famous Krein-Rutman theorem. They also introduced a simple iterative process for finding a positive eigenvector with positive eigenvalue of a strongly increasing and positively homogeneous mapping $T$ defined on a Banach space. Recently, various iterative processes have been extensively investigated for eigenvector problems and their related applications; see, e.g., [8, 10, 11, 12, 13].

In [14], Cai et al. provided the existence and uniqueness conditions for the solvability of an algebraic eigenvalue problem with eigenvector nonlinearity. They stated a plain self-consistent field iteration for solving nonlinear eigenvalue problem and then established local and global convergence results for the self-consistent field iteration.

In the study of eigenvector problems, we mainly deal with some sort of inequalities and equilibrium problems. One of the useful tools is to solve these problems is the KKM mappings and the KKM principle. Therefore, KKM theory plays a key role in studying the existence of solutions. For more information regarding these topics, we refer to $[15,16,17,18,19,20]$. In this paper, we continue these lines by replacing the identity function with a new function and we call our problems generalized common quasi-eigenvector problems.

\section{PRELiminaries}

Definition 2.1. Let $(Y,\|\|$.$) be a normed linear space with origin \theta$ over the field $\mathscr{K}$, the field of real numbers $\mathbb{R}$ or the field of complex numbers $\mathbb{C}$, and let $X$ be a nonempty subset of $Y$. Let $f: X \rightarrow Y$ and $g: X \rightarrow Y$ be two maps.

(a) The generalized common quasi-eigenvector problem (I) (in short [GCQEIVP]-(I)) is the following problem:

- Find $x_{0} \in X$ and $\lambda \in \mathscr{K}$ such that $f\left(x_{0}\right)=\lambda g\left(x_{0}\right)$.

In [GCQEIVP]-(I), $\lambda$ and $x_{0}$ are called eigenvalue and the corresponding weak quasieigenvector for $f$ with respect to $g$, respectively.

(b) The generalized common quasi-eigenvector problem (II) (in short [GCQEIVP]-(II)) is the following problem:

- Find $x_{0} \in X$ with $x_{0} \neq \theta$ and $\lambda \in \mathscr{K}$ such that $f\left(x_{0}\right)=\lambda g\left(x_{0}\right)$.

In [GCQEIVP]-(II), $\lambda$ and $x_{0}$ are called eigenvalue and the corresponding (strong) quasi-eigenvector for $f$ with respect to $g$, respectively.

(c) The generalized common quasi-eigenvector problem (III) (in short [GCQEIVP]-(III)) is the following problem:

- Find $x_{0} \in X$ and $\lambda \in \mathscr{K}$ such that $\lambda f\left(x_{0}\right)=g\left(x_{0}\right)$.

In [GCQEIVP]-(III), $\lambda$ and $x_{0}$ are called quasi-eigenvalue and the corresponding weak quasi-eigenvector for $f$ with respect to $g$, respectively.

(d) The generalized common quasi-eigenvector problem (IV) (in short [GCQEIVP]-(IV)) is the following problem:

- Find $x_{0} \in X$ with $x_{0} \neq \theta$ and $\lambda \in \mathscr{K}$ with $\lambda \neq 0$ such that $f\left(x_{0}\right)=\lambda g\left(x_{0}\right)$.

In [GCQEIVP]-(IV), $\lambda$ and $x_{0}$ are called eigenvalue and the corresponding (strong) quasi-eigenvector for $f$ with respect to $g$, respectively. [GCQEIVP]-(IV) can be stated as the following equivalent form:

- Find $x_{0} \in X$ with $x_{0} \neq \theta$ and $\lambda \in \mathscr{K}$ with $\lambda \neq 0$ such that $\lambda f\left(x_{0}\right)=g\left(x_{0}\right)$. 
It is easy to see that every solution of $[G C Q E I V P]-(I I)$ and $[G C Q E I V P]-(I V)$ is a solution of $[G C Q E I V P]-(I)$, and every solution of $[G C Q E I V P]-(I V)$ is a solution of $[G C Q E I V P]-(I I)$ and $[G C Q E I V P]-(I I I)$. While, the reverse implication does not hold.

Example 2.1. Let $\Omega$ be a bounded domain in $\mathbb{R}^{n}, n \geq 2$, with Lipschitz continuous boundary, and $1<p<\infty$. It is well known that the $p-$ Laplace operator on the domain $\Omega$ is defined by

$$
-\Delta_{p} u=-\operatorname{div}\left(|\nabla u|^{p-2} \nabla u\right)
$$

acting from the Sobolev space $X=W_{0}^{1, p}(\Omega)$ to its dual $X^{*}=W_{0}^{-1, p^{\prime}}(\Omega)$, where $p^{\prime}=\frac{p}{p-1}$. The nonlinear eigenvalue problem with Dirichlet boundary condition is

$$
\begin{aligned}
\Delta_{p} u & =\lambda\left(|u|^{p-2} u\right), & \text { in } \Omega, \\
u & =0, & \text { on } \partial \Omega .
\end{aligned}
$$

This operator finds a number of real fields in applied mathematics and mechanics; see, e.g, [21]. If $p=2$, then this problem simply reduces to the linear eigenvalue problem for the Laplace operator $-\triangle$, which has been studied extensively in the last 150 years.

If we denote by $f$ the differential operator defined by $-\triangle_{p}$ in the weak form, i.e.,

$$
\langle f(u), v\rangle=\int_{\Omega}|\nabla u(x)|^{p-2} \nabla u(x) \nabla v(x) d x, \quad\left(u \in W_{0}^{1, p}(\Omega)\right),
$$

and by $g$ the Nemytskij operator $[22,23,24,25]$ generated by the nonlinearity on the right hand side of (2.1), also in weak form, i.e.,

$$
\langle g(u), v\rangle=\int_{\Omega}|u(x)|^{p-2} u(x) v(x) d x, \quad\left(u \in W_{0}^{1, p}(\Omega)\right),
$$

then we obtain two operators acting from $X$ to its dual $X^{*}$. Here, the norm on $X$ is given by

$$
\|u\|=\left(\int_{\Omega}|\nabla u(x)|^{p} d x\right)^{\frac{1}{p}}
$$

which is, by the classical Poincare inequality $[26,27]$, equivalent to the usual norm on $X$ involving the $L_{p}-$ norm of $u$, as well. In this way, eigenvalue problem (2.1) may be rewritten for $\lambda \neq 0$ and $\mu=\frac{1}{\lambda}$, equivalently, as the operator equation

$$
f(u)=\mu g(u),
$$

which is a nonlinear eigenvalue problem for operator pair $(f, g)$. This problem is a special case of our problem whenever $X$ is a Hilbert space, for example $X=\mathbb{R}^{n}, n \geq 2$. A survey of methods and results for such problems can be found in [28].

In what follows, let $\mathscr{R}: Y \multimap Y$ be a set-valued mapping defined by

$$
\mathscr{R}(x)=\{r x: r \in \mathscr{K}\}, x \in Y .
$$

Clearly, for any $x \in Y, \mathscr{R}(x)$ is closed and convex. For any $u \in Y$ with $u \neq \theta, \mathscr{R}(u)$ can be regarded as the line in $Y$ passing through $\theta$ and $u$. The vertical cone $V C(X)$ of $X$ is defined by

$$
V C(X)=\bigcup_{x \in X} \mathscr{R}(x)
$$

see $[5,23]$. 
Remark 2.1. If we take $g$ as the identity mapping, then the generalized quasi-eigenvector problems reduces to the quasi-eigenvector problems studied by Lin and $\mathrm{Du}$ [6].

We use the technique of generalized KKM mappings and generalized KKM principle due to Ding (see, [20]).

Definition 2.2. Let $X$ be a nonempty subset of a topological vector space $E$. A set-valued mapping $F: X \multimap E$ is said to be a generalized KKM mapping (GKKM) if for each nonempty finite set $\left\{x_{1}, \ldots, x_{n}\right\} \subseteq X$, there exists a set $\left\{y_{1}, \ldots, y_{n}\right\}$ of points of $E$, not necessarily all different, such that for each subset $\left\{y_{i_{1}}, \ldots, y_{i_{k}}\right\}$ of $\left\{y_{1}, \ldots, y_{n}\right\}$, we have

$$
\operatorname{conv}\left\{y_{i_{1}}, \ldots, y_{i_{k}}\right\} \subseteq \bigcup_{j=1}^{k} F\left(x_{i_{j}}\right) .
$$

The following theorem is a particular case of Corollary 3.1 in [20].

Theorem 2.1. Let $X$ be a nonempty subset of a topological vector space $E$, and $F: X \multimap E$ be a GKKM mapping. Assume that there exists a nonempty compact convex subset $C$ of $X$ such that $K=\bigcap_{x \in C} F(x)$ is compact. Then,

$$
\bigcap_{x \in X} F(x) \neq \emptyset
$$

Let $A$ be a nonempty set of a normed linear space. We denote by $\langle A\rangle, \bar{A}, \partial A$, and int $A$, the family of all nonempty finite subsets of $X$, closure of $A$, boundary of $A$, and interior of $A$, respectively.

\section{MAIN RESUlTS}

The following result which plays a key role in the proof of the main results is a generalization of Theorem 3.2 in [6].

Theorem 3.1. Let $(Y,\|\|$.$) be a normed linear space with origin \theta$ and $X$ be a nonempty subset of $Y$. Let $f: Y \rightarrow Y$ and $g: Y \rightarrow Y$ be continuous mappings such that $g(x)=\theta$ implies that $f(x)=\theta$. Then, for each $y \in Y$,

$$
G(y)=\{x \in X: d(f(y), \mathscr{R}(g(x))) \geq d(f(x), \mathscr{R}(g(x)))\}
$$

is a closed set in $X$.

Proof. We follow the proof of Theorem 3.2 in [6]. Let $\hat{x} \in \overline{G(y)}$. Then there exists a sequence $\left(x_{n}\right)$ in $G(y)$ such that $x_{n} \rightarrow \hat{x}$ as $n \rightarrow \infty$. If $g(\hat{x})=\theta$, then $f(\hat{x})=\theta$ and so $\hat{x} \in G(y)$. If $g(\hat{x}) \neq \theta$, then $\|g(\hat{x})\|>0$. Since $g\left(x_{n}\right) \rightarrow g(\hat{x})$, without loss of generality, we may assume that $g\left(x_{n}\right) \neq \theta$ and $\left\|g\left(x_{n}\right)-g(\hat{x})\right\|<\|g(\hat{x})\|$, for all $n \in N$. There exist $v \in \mathscr{R}(g(\hat{x}))$ and $z_{n} \in \mathscr{R}\left(g\left(x_{n}\right)\right)$ such that

$$
\|f(\hat{x})-v\|=d(f(\hat{x}), \mathscr{R}(g(\hat{x}))),
$$

and

$$
\left\|f(\hat{x})-z_{n}\right\|=d\left(f(\hat{x}), \mathscr{R}\left(g\left(x_{n}\right)\right)\right),
$$


for all $n \in \mathbb{N}$. We show that, for all $n \in N$, the following inequality holds

$$
\begin{aligned}
& d(f(\hat{x}), \mathscr{R}(g(\hat{x}))) \leq d\left(f\left(x_{n}\right), \mathscr{R}\left(g\left(x_{n}\right)\right)\right)+\left\|f(\hat{x})-f\left(x_{n}\right)\right\| \\
& \quad+\frac{\|f(\hat{x})\|+\|f(\hat{x})-v\|}{\|g(\hat{x})\|-\left\|g\left(x_{n}\right)-g(\hat{x})\right\|}\left\|g\left(x_{n}\right)-g(\hat{x})\right\| .
\end{aligned}
$$

Since there exists $w_{n} \in \mathscr{R}\left(g\left(x_{n}\right)\right)$ such that

$$
\left\|f\left(x_{n}\right)-w_{n}\right\|=d\left(f\left(x_{n}\right), \mathscr{R}\left(g\left(x_{n}\right)\right)\right),
$$

we have from (3.2) and (3.4) that

$$
\begin{aligned}
\left\|f(\hat{x})-z_{n}\right\| & =d\left(f(\hat{x}), \mathscr{R}\left(g\left(x_{n}\right)\right)\right) \\
& \leq\left\|f(\hat{x})-w_{n}\right\| \\
& \leq\left\|f(\hat{x})-f\left(x_{n}\right)\right\|+d\left(f\left(x_{n}\right), \mathscr{R}\left(g\left(x_{n}\right)\right)\right),
\end{aligned}
$$

for all $n \in \mathbb{N}$. If $\|f(\hat{x})-v\| \leq\left\|f(\hat{x})-z_{n}\right\|$, then by (3.1) and (3.5), we obtain

$$
\begin{aligned}
d(f(\hat{x}), \mathscr{R}(g(\hat{x}))) & =\|f(\hat{x})-v\| \\
& \leq\left\|f(\hat{x})-z_{n}\right\| \\
& \leq\left\|f(\hat{x})-f\left(x_{n}\right)\right\|+d\left(f\left(x_{n}\right), \mathscr{R}\left(g\left(x_{n}\right)\right)\right) .
\end{aligned}
$$

Hence, (3.3) holds in this case. If $\|f(\hat{x})-v\|>\left\|f(\hat{x})-z_{n}\right\|$, then $z_{n} \neq \theta$. Indeed, if $z_{n}=\theta$, then

$$
\|f(\hat{x})-v\|=d(f(\hat{x}), \mathscr{R}(g(\hat{x}))) \leq\left\|f(\hat{x})-z_{n}\right\|,
$$

which leads to a contradiction. Thus, $z_{n} \neq \theta$. Since, $z_{n} \in \mathscr{R}\left(g\left(x_{n}\right)\right)$, we see that there exists $t_{n} \in \mathscr{K}$ such that $z_{n}=t_{n} g\left(x_{n}\right)$. Then,

$$
\left|t_{n}\right|=\frac{\left\|z_{n}\right\|}{\left\|g\left(x_{n}\right)\right\|}>0
$$

Let $v_{n}=t_{n} g(\hat{x}) \in \mathscr{R}(g(\hat{x}))$. Since

$$
\left\|z_{n}\right\| \leq\|f(\hat{x})\|+\left\|f(\hat{x})-z_{n}\right\|<\|f(\hat{x})\|+\|f(\hat{x})-v\|,
$$

we have

$$
\begin{aligned}
\left\|v_{n}-z_{n}\right\| & =\frac{\left\|z_{n}\right\|}{\left\|g\left(x_{n}\right)\right\|}\left\|g(\hat{x})-g\left(x_{n}\right)\right\| \\
& <\frac{\|f(\hat{x})\|+\|f(\hat{x})-v\|}{\left\|g\left(x_{n}\right)\right\|}\left\|g(\hat{x})-g\left(x_{n}\right)\right\| \\
& \leq \frac{\|f(\hat{x})\|+\|f(\hat{x})-v\|}{\|g(\hat{x})\|-\left\|g(\hat{x})-g\left(x_{n}\right)\right\|}\left\|g(\hat{x})-g\left(x_{n}\right)\right\| .
\end{aligned}
$$

Combining (3.1), (3.2), (3.5) and (3.7), we get our claim (3.3) as follows:

$$
\begin{aligned}
& d(f(\hat{x}), \mathscr{R}(g(\hat{x}))) \leq\left\|f(\hat{x})-v_{n}\right\| \\
& \quad \leq\left\|f(\hat{x})-z_{n}\right\|+\left\|z_{n}-v_{n}\right\| \\
& \quad<d\left(f(\hat{x}), \mathscr{R}\left(g\left(x_{n}\right)\right)\right)+\frac{\|f(\hat{x})\|+\|f(\hat{x})-v\|}{\|g(\hat{x})\|-\left\|g\left(x_{n}\right)-g(\hat{x})\right\|}\left\|g\left(x_{n}\right)-g(\hat{x})\right\| \\
& \quad \leq d\left(f\left(x_{n}\right), \mathscr{R}\left(g\left(x_{n}\right)\right)\right)+\left\|f(\hat{x})-f\left(x_{n}\right)\right\|+\frac{\|f(\hat{x})\|+\|f(\hat{x})-v\|}{\|g(\hat{x})\|-\left\|g\left(x_{n}\right)-g(\hat{x})\right\|}\left\|g\left(x_{n}\right)-g(\hat{x})\right\| .
\end{aligned}
$$


Next, we take $a \in \mathscr{R}(g(\hat{x}))$ and $u_{n} \in \mathscr{R}\left(g\left(x_{n}\right)\right)$ such that

$$
\|f(y)-a\|=d(f(y), \mathscr{R}(g(\hat{x})))
$$

and

$$
\left\|f(y)-u_{n}\right\|=d\left(f(y), \mathscr{R}\left(g\left(x_{n}\right)\right)\right),
$$

for all $n \in \mathbb{N}$. We also claim that the following inequality holds, for all $n \in N$,

$$
d\left(f(y), \mathscr{R}\left(g\left(x_{n}\right)\right)\right) \leq\|f(y)-a\|+\frac{\|a\|}{\|g(\hat{x})\|}\left\|g\left(x_{n}\right)-g(\hat{x})\right\| .
$$

If $a=\theta$, then we have from (3.9) that

$$
\left\|f(y)-u_{n}\right\|=d\left(f(y), \mathscr{R}\left(g\left(x_{n}\right)\right)\right) \leq\|f(y)-a\| .
$$

So, (3.10) holds in this case. If $a \neq \theta$, then $a=\gamma g(\hat{x})$ for some $\gamma \neq 0$. Thus,

$$
|\gamma|=\frac{\|a\|}{\|g(\hat{x})\|}>0 .
$$

For each $n \in \mathbb{N}$, let $c_{n}=\gamma g\left(x_{n}\right) \in \mathscr{R}\left(g\left(x_{n}\right)\right)$. Consequently, it follows that

$$
\begin{aligned}
d\left(f(y), \mathscr{R}\left(g\left(x_{n}\right)\right)\right) & \leq\left\|f(y)-c_{n}\right\| \\
& \leq\|f(y)-a\|+\left\|a-c_{n}\right\| \\
& =\|f(y)-a\|+\frac{\|a\|}{\|g(\hat{x})\|}\left\|g\left(x_{n}\right)-g(\hat{x})\right\|,
\end{aligned}
$$

which proves (3.10). Since $x_{n} \in G(y)$, we have

$$
d\left(f(y), \mathscr{R}\left(g\left(x_{n}\right)\right)\right) \geq d\left(f\left(x_{n}\right), \mathscr{R}\left(g\left(x_{n}\right)\right)\right) .
$$

From (3.3), (3.8), (3.10) and (3.11), we obtain

$$
\begin{aligned}
d(f(y), \mathscr{R}(g(\hat{x}))) & =\|f(y)-a\| \\
& \geq d\left(f(y), \mathscr{R}\left(g\left(x_{n}\right)\right)\right)-\frac{\|a\|}{\|g(\hat{x})\|}\left\|g\left(x_{n}\right)-g(\hat{x})\right\| \\
& \geq d\left(f\left(x_{n}\right), \mathscr{R}\left(g\left(x_{n}\right)\right)\right)-\frac{\|a\|}{\|g(\hat{x})\|}\left\|g\left(x_{n}\right)-g(\hat{x})\right\| \\
& \geq d(f(\hat{x}), \mathscr{R}(g(\hat{x})))-\left\|f(\hat{x})-f\left(x_{n}\right)\right\| \\
& -\frac{\|f(\hat{x})\|+\|f(\hat{x})-v\|}{\|g(\hat{x})\|-\left\|g\left(x_{n}\right)-g(\hat{x})\right\|}\left\|g\left(x_{n}\right)-g(\hat{x})\right\| \\
& -\frac{\|a\|}{\|g(\hat{x})\|}\left\|g\left(x_{n}\right)-g(\hat{x})\right\|,
\end{aligned}
$$

for all $n \in \mathbb{N}$. Since $f$ and $g$ are continuous and $x_{n} \rightarrow \hat{x}$ as $n \rightarrow \infty$, we conclude from (3.12) that

$$
d(f(y), \mathscr{R}(g(\hat{x}))) \geq d(f(\hat{x}), \mathscr{R}(g(\hat{x})))
$$

which means that $\hat{x} \in G(y)$. This completes the proof.

Theorem 3.2. Let $(Y,\|\|$.$) be a normed linear space with origin \theta, X$ be a nonempty subset of $Y$. Let $f: Y \rightarrow Y$ be a one to one, onto continuous map and $g: Y \rightarrow Y$ be a continuous map such that $g(x)=\theta$ implies that $f(x)=\theta$. Suppose that 
$\left(H_{1}\right)$ there exists a nonempty compact subset $K$ of $X$ and a nonempty compact convex subset $C$ of $X$ such that for each $y \in X \backslash K$ there exists $z \in C$ such that $d(f(z), \mathscr{R}(g(y)))<$ $d(f(y), \mathscr{R}(g(y)))$.

Then, there exists $x_{0} \in X$ such that

$$
d\left(f(x), \mathscr{R}\left(g\left(x_{0}\right)\right)\right) \geq d\left(f\left(x_{0}\right), \mathscr{R}\left(g\left(x_{0}\right)\right)\right),
$$

for all $x \in X$. Moreover, if $f\left(x_{0}\right) \notin \mathscr{R}\left(g\left(x_{0}\right)\right)$, then $f\left(x_{0}\right)$ must be a boundary point of $f(X)$.

Proof. Define a multifunction $G: X \multimap X$ by

$$
G(y)=\{x \in X: d(f(y), \mathscr{R}(g(x))) \geq d(f(x), \mathscr{R}(g(x)))\} .
$$

By Theorem 3.1, $G(y)$ is closed for each $y \in X$. We show that $f \circ G: X \multimap Y$ is a generalized KKM mapping. Suppose that $y_{1}, \ldots, y_{n}$ are in $X$ and $y_{0} \notin \bigcup_{k=1}^{n} G\left(y_{k}\right)$. Then, we have

$$
d\left(f\left(y_{0}\right), \mathscr{R}\left(g\left(y_{0}\right)\right)\right)>d\left(f\left(y_{k}\right), \mathscr{R}\left(g\left(y_{0}\right)\right)\right), k=1,2, \ldots, n .
$$

Let

$$
S\left(y_{0}\right)=\left\{y \in X: d\left(f\left(y_{0}\right), \mathscr{R}\left(g\left(y_{0}\right)\right)\right)>d\left(f(y), \mathscr{R}\left(g\left(y_{0}\right)\right)\right)\right\} .
$$

Clearly, $y_{k} \in S\left(y_{0}\right)$, for each $1 \leq k \leq n$. We take $z_{k} \in \mathscr{R}\left(g\left(y_{0}\right)\right)$ such that

$$
d\left(f\left(y_{0}\right), \mathscr{R}\left(g\left(y_{0}\right)\right)\right)>\left\|f\left(y_{k}\right)-z_{k}\right\| .
$$

Let $\lambda_{k} \geq 0$ and $\sum_{k=1}^{n} \lambda_{k}=1$. Then, we have

$$
\begin{aligned}
\left\|\sum_{k=1}^{n} \lambda_{k} f\left(y_{k}\right)-\sum_{k=1}^{n} \lambda_{k} z_{k}\right\| & \leq \max _{1 \leq k \leq n}\left\|f\left(y_{k}\right)-z_{k}\right\| \\
& <d\left(f\left(y_{0}\right), \mathscr{R}\left(g\left(y_{0}\right)\right)\right) .
\end{aligned}
$$

Since $\mathscr{R}\left(g\left(y_{0}\right)\right)$ is convex

$$
d\left(\sum_{k=1}^{n} \lambda_{k} f\left(y_{k}\right), \mathscr{R}\left(g\left(y_{0}\right)\right)\right)<d\left(f\left(y_{0}\right), \mathscr{R}\left(g\left(y_{0}\right)\right)\right) .
$$

Hence, we deduce that

$$
f^{-1}\left(\operatorname{conv}\left\{f\left(y_{1}\right), \ldots, f\left(y_{n}\right)\right\}\right) \subseteq S\left(y_{0}\right) .
$$

Since $y_{0} \notin S\left(y_{0}\right)$, we have $y_{0} \notin f^{-1}\left(\operatorname{conv}\left\{f\left(y_{1}\right), \ldots, f\left(y_{n}\right)\right\}\right)$. Consequently,

$$
f^{-1}\left(\operatorname{conv}\left\{f\left(y_{1}\right), \ldots, f\left(y_{n}\right)\right\}\right) \subseteq \bigcup_{k=1}^{n} G\left(y_{k}\right) .
$$

Since $f$ is onto, we get

$$
\operatorname{conv}\left\{f\left(y_{1}\right), \ldots, f\left(y_{n}\right)\right\} \subseteq \bigcup_{k=1}^{n} f \circ G\left(y_{k}\right) .
$$

This shows that $f \circ G$ is a generalized KKM mapping. Letting $K=\bigcap_{x \in C} G(x)$, thanks to injectivity of $f$, we deduce that $f(K)=\bigcap_{x \in C} f \circ G(x)$. Condition $\left(H_{1}\right)$ implies that $K$ is compact. Hence, by Theorem 2.1, $\bigcap_{x \in X} f \circ G(x) \neq \emptyset$. Therefore, $\bigcap_{x \in X} G(x) \neq \emptyset$. Let $x_{0} \in \bigcap_{x \in X} G(x)$. Then

$$
d\left(f(x), \mathscr{R}\left(g\left(x_{0}\right)\right)\right) \geq d\left(f\left(x_{0}\right), \mathscr{R}\left(g\left(x_{0}\right)\right)\right)
$$


for all $x \in X$. If $f\left(x_{0}\right) \notin \mathscr{R}\left(g\left(x_{0}\right)\right)$, then there exists $u_{0} \in \mathscr{R}\left(g\left(x_{0}\right)\right)$ such that

$$
d\left(f\left(x_{0}\right), u_{0}\right)=d\left(f\left(x_{0}\right), \mathscr{R}\left(g\left(x_{0}\right)\right)>0 .\right.
$$

Suppose that $f\left(x_{0}\right) \in \operatorname{int} f(X)$. Then there exists $t>0$, small enough, such that

$$
B\left(f\left(x_{0}\right), t\right) \subseteq \operatorname{int} f(X)
$$

and

$$
0<t<d\left(f\left(x_{0}\right), \mathscr{R}\left(g\left(x_{0}\right)\right)\right)
$$

It is clear that

$$
B\left(f\left(x_{0}\right), t\right) \bigcap B\left(u_{0}, d\left(f\left(x_{0}\right), \mathscr{R}\left(g\left(x_{0}\right)\right)\right)-t\right) \neq \emptyset .
$$

If $y=f(x), x \in X$, is any element of this intersection, then

$$
d\left(f(x), \mathscr{R}\left(g\left(x_{0}\right)\right)\right) \leq d\left(f(x), u_{0}\right)<d\left(f\left(x_{0}\right), \mathscr{R}\left(g\left(x_{0}\right)\right)\right)
$$

which is a contradiction.

Using Theorem 3.2, we have the following theorem which gives a sufficient condition for the existence of solution of problem $[G Q E I V P]-(I)$.

Theorem 3.3. Let $(Y,\|\|$.$) be a normed linear space with origin \theta, X$ be a nonempty subset of $Y$. Let $f: Y \rightarrow Y$ be a one to one, onto continuous map and $g: Y \rightarrow Y$ be a continuous map such that $g(x)=\theta$ implies that $f(x)=\theta$. Suppose that the condition $\left(H_{1}\right)$ in Theorem 3.2 holds. Assume that either $\operatorname{Ker} f \cap X \neq \emptyset$ or $f(X)$ is an open subset of $Y$. Then, there exist $x_{0} \in X$ and $\lambda \in \mathscr{K}$ such that $f\left(x_{0}\right)=\lambda g\left(x_{0}\right)$.

Proof. We first assume that $\operatorname{Ker} f \cap X \neq \emptyset$. By Theorem 3.2, there exists $x_{0} \in X$ such that $d\left(f(x), \mathscr{R}\left(g\left(x_{0}\right)\right)\right) \geq d\left(f\left(x_{0}\right), \mathscr{R}\left(g\left(x_{0}\right)\right)\right)$, for all $x \in X$. Since $\operatorname{Ker} f \cap X \neq \emptyset$, there exists $v \in X$ such that $f(v)=0$. Therefore,

$$
d\left(f\left(x_{0}\right), \mathscr{R}\left(g\left(x_{0}\right)\right)\right) \leq d\left(f(v), \mathscr{R}\left(g\left(x_{0}\right)\right)\right)=0 .
$$

Thus, we have $d\left(f\left(x_{0}\right), \mathscr{R}\left(g\left(x_{0}\right)\right)\right)=0$ or $f\left(x_{0}\right) \in \mathscr{R}\left(g\left(x_{0}\right)\right)$. So there exists $\lambda \in \mathscr{K}$ such that $f\left(x_{0}\right)=\lambda g\left(x_{0}\right)$. Now suppose that $f(X)$ is an open subset of $Y$ and $f\left(x_{0}\right) \notin \mathscr{R}\left(g\left(x_{0}\right)\right)$. Then, by Theorem 3.2, $f\left(x_{0}\right)$ is a boundary point of $f(X)$, which is a contradiction.

If we take $g=I$, we get the following corollary which gives an existence result for eigenvector problem. Note that our sufficient conditions for the solution of eigenvector problem are different from Theorem 3.5 in [6].

Corollary 3.1. Let $(Y,\|\|$.$) be a normed linear space with origin \theta, X$ be a nonempty subset of $Y$. Let $f: Y \rightarrow Y$ be a one to one, onto continuous map satisfying $f(\theta)=\theta$. Suppose that the following condition holds.

$\left(C_{1}\right)$ there exist a nonempty compact subset $K$ of $X$ and a nonempty compact convex subset $C$ of $X$ such that for each $y \in X \backslash K$ there exists $z \in C$ such that $d(f(z), \mathscr{R}(y))<$ $d(f(y), \mathscr{R}(y))$.

Assume that either Kerf $\cap X \neq \emptyset$ or $f(X)$ is an open subset of $Y$. Then, there exist $x_{0} \in X$ and $\lambda \in \mathscr{K}$ such that $f\left(x_{0}\right)=\lambda x_{0}$.

The following existence theorem related to problem $[G Q E I V P]-(I I)$ is established immediately from Theorem 3.3. 
Theorem 3.4. Under the same hypothesis of Theorem 3.3 and assume that $\theta \notin X$, problem $[G Q E I V P]-(I I)$ has a solution.

We also have the following existence theorem for solution of problem $[G Q E I V P]-(I I)$.

Theorem 3.5. Let $(Y,\|\|$.$) be a normed linear space with origin \theta, X$ be a nonempty subset of $Y$. Let $f: Y \rightarrow Y$ be a one to one, onto continuous map and $g: Y \rightarrow Y$ be a continuous map satisfying that $g(x)=\theta$ implies $f(x)=\theta, \theta \notin X \cup g(X)$, and $g(X) \subseteq V C(f(X))$. Suppose that the condition $\left(H_{1}\right)$ in Theorem 3.2 holds. Then, there exist $x_{0} \in X$ with $x_{0} \neq \theta$ and $\lambda \in \mathscr{K}$ such that $f\left(x_{0}\right)=\lambda g\left(x_{0}\right)$.

Proof. By Theorem 3.2, there is $\theta \neq x_{0} \in X$ such that $d\left(f(x), \mathscr{R}\left(g\left(x_{0}\right)\right)\right) \geq d\left(f\left(x_{0}\right), \mathscr{R}\left(g\left(x_{0}\right)\right)\right)$, for all $x \in X$. Since $g(X) \subseteq V C(f(X))$, there exists $x_{1} \in X$ such that $g\left(x_{0}\right) \in \mathscr{R}\left(f\left(x_{1}\right)\right)$. Thus, there exists $\gamma \in \mathscr{K}$ such that $g\left(x_{0}\right)=\gamma f\left(x_{1}\right)$. Since $\theta \notin g(X)$, we have $\gamma \neq 0$. Hence, $f\left(x_{1}\right)=$ $\gamma^{-1} g\left(x_{0}\right) \in \mathscr{R}\left(g\left(x_{0}\right)\right)$. It follows that

$$
d\left(f\left(x_{0}\right), \mathscr{R}\left(g\left(x_{0}\right)\right)\right) \leq d\left(f\left(x_{1}\right), \mathscr{R}\left(g\left(x_{0}\right)\right)\right)=0,
$$

and we have $d\left(f\left(x_{0}\right), \mathscr{R}\left(g\left(x_{0}\right)\right)\right)=0$ or $f\left(x_{0}\right) \in \mathscr{R}\left(g\left(x_{0}\right)\right)$. Therefore, there exists $\lambda \in \mathscr{K}$ such that $f\left(x_{0}\right)=\lambda g\left(x_{0}\right)$.

Remark 3.1. Under the same of hypothesis of Theorem 3.5 and the assumption $\theta \notin f(X)$, we get a solution of the problem $[G Q E I V P]-(I V)$.

By interchanging the roles of $f$ and $g$ in Theorem 3.3 and Theorem 3.5, we obtain the following existence theorems for solutions of problems $[C Q E I V P]-(I I I)$ and $[C Q E I V P]-(I V)$, respectively.

Theorem 3.6. Let $(Y,\|\|$.$) be a normed linear space with origin \theta, X$ be a nonempty subset of $Y$. Let $f: Y \rightarrow Y$ be a continuous map and $g: Y \rightarrow Y$ be a one to one, onto continuous map such that $f(x)=\theta$ implies that $g(x)=\theta$. Assume that either $\operatorname{Ker}(g) \cap X \neq \emptyset$ or $g(X)$ is an open subset of $Y$. Suppose that

$\left(\mathrm{H}_{2}\right)$ there exist a nonempty compact subset $K$ of $X$ and a nonempty compact convex subset $C$ of $X$ such that for each $y \in X \backslash K$ there exists $z \in C$ such that $d(g(z), \mathscr{R}(f(y)))<$ $d(g(y), \mathscr{R}(f(y)))$.

Then, there exist $x_{0} \in X$ and $\lambda \in \mathscr{K}$ such that $\lambda f\left(x_{0}\right)=g\left(x_{0}\right)$.

Theorem 3.7. Let $(Y,\|\|$.$) be a normed linear space with origin \theta, X$ be a nonempty subset of $Y$ with $\theta \notin X$. Let $f: Y \rightarrow Y$ be a continuous map. Let $g: Y \rightarrow Y$ be a one to one, onto continuous map satisfying $f(x)=\theta \Rightarrow g(x)=\theta$, and $\theta \notin f(X) \subseteq V C(g(X))$. Suppose that the condition $\left(H_{2}\right)$ in Theorem 3.6 holds. Then, there exist $x_{0} \in X$ with $x_{0} \neq \theta$ and $\lambda \in \mathscr{K}$ with $\lambda \neq 0$ such that $\lambda f\left(x_{0}\right)=g\left(x_{0}\right)$.

The following corollary which improves Theorem 4.2 in [6], is established immediately from Theorem 3.6 by letting $g=I$.

Corollary 3.2. Let $(Y,\|\|$.$) be a normed linear space with origin \theta, X$ be a nonempty open subset of $Y$. Let $f: Y \rightarrow Y$ be a continuous map with $f^{-1}(\theta) \subseteq\{\theta\}$. Suppose that

$\left(C_{2}\right)$ there exist a nonempty compact subset $K$ of $X$ and a nonempty compact convex subset $C$ of $X$ such that for each $y \in X \backslash K$ there exists $z \in C$ such that $d(z, \mathscr{R}(f(y)))<$ $d(y, \mathscr{R}(f(y)))$. 
Then, there exist $x_{0} \in X$ and $\lambda \in \mathscr{K}$ such that $\lambda f\left(x_{0}\right)=x_{0}$.

Corollary 3.3. Let $J$ be an arbitrary index set. Let $\left(Y_{j},\|\cdot\|_{j}\right)$, for $j \in J$, be a normed linear space with origin $\theta_{j}, X_{j}$ be a nonempty subset of $Y_{j}$. Let $Y=\prod_{j \in J} Y_{j}$ with origin $\theta=\left(\theta_{j}\right)_{j \in J}$ and $X=\prod_{j \in J} X_{j}$. For $j \in J$, let $f_{j}: Y \rightarrow Y_{j}$ be a one to one, onto continuous map and $g_{j}: Y \rightarrow Y_{j}$ be a continuous map such that $g_{j}(x)=\theta_{j}$ implies that $f_{j}(x)=\theta_{j}$. Assume that $\operatorname{Ker}_{j} \cap X \neq \emptyset$. Define $f: Y \rightarrow Y$ by $f(x)=\left(f_{j}(x)\right)_{j \in J}$ and $g: Y \rightarrow Y$ by $g(x)=\left(g_{j}(x)\right)_{j} \in J$. Suppose that the condition $\left(H_{1}\right)$ in Theorem 3.2 holds. Then, there exist $x_{0} \in X$ and $\lambda \in \mathscr{K}$ such that $f\left(x_{0}\right)=\lambda g\left(x_{0}\right)$.

Proof. Clearly, $Y$ is a normed linear space with norm $\|y\|=\sup _{j \in J}\left\|y_{j}\right\|$ where $y=\left(y_{j}\right)_{j \in J} \in Y$, and $X$ is a nonempty subset of $Y$. Moreover, $f$ is an one to one, onto continuous map and $g$ is a continuous map such that $g(x)=\theta$ implies that $f(x)=\theta$. All conditions of Theorem 3.2 are satisfied, and hence there exists $x_{0} \in X$ such that $d\left(f(x), \mathscr{R}\left(g\left(x_{0}\right)\right)\right) \geq d\left(f\left(x_{0}\right), \mathscr{R}\left(g\left(x_{0}\right)\right)\right)$, for all $x \in X$. Since $\operatorname{Ker} f_{j} \cap X \neq \emptyset$, then there exists $v \in X$ such that $f_{j}(v)=\theta_{j}$ and hence $f(v)=\theta$. Therefore, $d\left(f\left(x_{0}\right), \mathscr{R}\left(g\left(x_{0}\right)\right)\right) \leq d\left(f(v), \mathscr{R}\left(g\left(x_{0}\right)\right)\right)=0$ implies that $d\left(f\left(x_{0}\right), \mathscr{R}\left(g\left(x_{0}\right)\right)\right)=0$, and hence $\left.f\left(x_{0}\right) \in \mathscr{R}\left(g\left(x_{0}\right)\right)\right)$. Thus, there exists $\lambda \in \mathscr{K}$ such that $f\left(x_{0}\right)=\lambda g\left(x_{0}\right)$.

Corollary 3.4. Let $J$ be an arbitrary index set. Let $\left(Y_{j},\|\cdot\|_{j}\right)$, for $j \in J$, be a normed linear space with origin $\theta_{j}, X_{j}$ be a nonempty subset of $Y_{j}$ with $\theta_{j} \notin X_{j}$. Let $Y=\prod_{j \in J} Y_{j}$ with origin $\theta=\left(\theta_{j}\right)_{j \in J}$ and $X=\prod_{j \in J} X_{j}$. For $j \in J$, Let $f_{j}: Y \rightarrow Y_{j}$ be a one to one, onto continuous map and $g_{j}: Y \rightarrow Y_{j}$ be a continuous map such that $g_{j}(x)=\theta_{j}$ implies that $f_{j}(x)=\theta_{j}, \theta_{j} \notin$ $X_{j} \cup g\left(X_{j}\right)$. Define $f: Y \rightarrow Y$ by $f(x)=\left(f_{j}(x)\right)_{j \in J}$ and $g: Y \rightarrow Y$ by $g(x)=\left(g_{j}(x)\right)_{j} \in J$. Assume that $g(X) \subseteq V C(f(x))=\cup_{x \in X} \mathscr{R}(f(x))$. Suppose that the condition $\left(H_{1}\right)$ in Theorem 3.2 holds. Then, there exist $x_{0} \in X$ and $\lambda \in \mathscr{K}$ such that $f\left(x_{0}\right)=\lambda g\left(x_{0}\right)$.

Proof. Clearly, $Y$ is a normed linear space with norm $\|y\|=\sup _{j \in I J}\left\|y_{j}\right\|$ where $y=\left(y_{j}\right)_{j \in J} \in Y$, and $X$ is a nonempty subset of $Y$. Moreover, $f$ is an one to one, onto continuous map and $g$ is a continuous map such that $g(x)=\theta$ implies that $f(x)=\theta$. All conditions of Theorem 3.2 are satisfied, and hence there exists $x_{0} \in X$ such that $d\left(f(x), \mathscr{R}\left(g\left(x_{0}\right)\right)\right) \geq d\left(f\left(x_{0}\right), \mathscr{R}\left(g\left(x_{0}\right)\right)\right)$, for all $x \in X$. Since $g(X) \subseteq V C(f(x))=\cup_{x \in X} \mathscr{R}(f(x))$, there exists $v \in X$ such that $g\left(x_{0}\right) \in$ $\mathscr{R}(f(v))$. Thus, there exists $\lambda \in \mathscr{K}$ such that $g\left(x_{0}\right)=\lambda f(v)$ and $\lambda \neq 0$. Thus, $f(v)=$ $\lambda^{-1} g\left(x_{0}\right) \in \mathscr{R}\left(g\left(x_{0}\right)\right.$ which follows that $d\left(f\left(x_{0}\right), \mathscr{R}\left(g\left(x_{0}\right)\right)\right) \leq d\left(f(v), \mathscr{R}\left(g\left(x_{0}\right)\right)\right)=0$. It implies that $\left.f\left(x_{0}\right) \in \mathscr{R}\left(g\left(x_{0}\right)\right)\right)=0$, and hence there exists $\lambda \in \mathscr{K}$ such that $f\left(x_{0}\right)=\lambda g\left(x_{0}\right)$.

\section{Acknowledgments}

The authors would like to thank the anonymous referees for their suggestions and comments which improved the paper greatly. The research of C.-F. Wen was funded by Ministry of Science and Technology, Taiwan under grant number 109-2115-M-037-001.

\section{REFERENCES}

[1] D. Shemsh, Common eigenvectors of two matrices, Linear Alg. Appl. 62 (1984), 11-18.

[2] A. George, K. D. Ikramov, Common invariant subspaces of two matrices, Linear Algebra and its Application. 287 (1999), 171-179.

[3] J. Li, Some eigenvector theorems proved by a Fan-KKM theorem, J. Math. Anal. Appl. 263 (2001), $738-747$.

[4] F. Fan, A generalization of Tykhonoff's fixed point theorem, Math. Ann. 142 (1961), 305-310.

[5] J. Li, S. Park, On solution of generalized complementarity and eigenvector problems, Nonlinear Anal. 65 (2006), 12-24. 
[6] L. J. Lin, W. S. Du, On common quasi-eigenvector problems, Nonlinear Anal. 69 (2008), 463-471.

[7] R. Abreu, C. Morales-Rodrigo, A. Suarez, Some eigenvalue problems with non-local boundary conditions and applications, Commun. Pure Appl. Anal. 13 (2014), 2465-2474.

[8] S. J. Hwang, M. D. Collins, S. N. Ravi, V. K. Ithapu, N. Adluru, S. C. Johnson, V. Singh, A projection free method for generalized eigenvalue problem with a nonsmooth regularizer, Proc IEEE Int Conf Comput Vis. 2015, (2015), 1841-1849.

[9] Y. Song, L. Qi, Positive eigenvalue-eigenvector of nonlinear positive mappings, Front. Math. China. 9 (2014), 181-199.

[10] S.Y. Cho, A monotone Bregan projection algorithm for fixed point and equilibrium problems in a reflexive Banach space, Filomat, 34 (2020), 1487-1497.

[11] T.H. Cuong, J.C. Yao, N.D. Yen, Qualitative properties of the minimum sum-of-squares clustering problem, Optimization, 69 (2020), 2131-2154.

[12] P. Cubiotti, J.C. Yao, On the Cauchy problem for a class of differential inclusions with applications, Appl. Anal. 99 (2020), 2543-2554.

[13] S.Y. Cho, X. Qin, On the strong convergence of an iterative process for asymptotically strict pseudocontractions and equilibrium problems, Appl. Math. Comput. 235 (2014), 430-438.

[14] Y. Cai, L. H. Zhang, Z. Bai, R. C. Li, On an eigenvector-dependent nonlinear eigenvalue problem, SIAM J. Matrix Anal. Appl. 39 (2018), 1360-1382.

[15] L. C. Zeng, S. Y. Wu, J. C. Yao, Generalized KKM theorem with applications to generalized minimax inequalities and generalized equilibrium problems, Taiwanese J. Math. 10 (2007), 1497-1514.

[16] M. Tavakoli, A.P. Farajzadeh, D. Inoan, On a generalized variational inequality problem, Filomat, 32 (2018), 2433-2441.

[17] O. Chadli, N. C. Wong, J. C. Yao, Equilibrium problems with applications to eigenvalue problems, J. Optim. Theory Appl. 117 (2003), 245-266.

[18] A. Farajzadeh, A. Shafie, On system of vector quasi-equilibrium problems for multi valued mappings, Kragujevac J. Math. 42 (2018), 357-369.

[19] P. Lohawech, A. Kaewcharoen, A. Farajzadeh, Algorithms for the common solution of the split variational inequality problems and fixed point problems with applications, J. Inequal. Appl. 2018 (2018), 358.

[20] X. P. Ding, Generalized G-KKM theorems in generalized convex spaces and their applications, J. Math. Anal. Appl. 266 (2002), 21-37.

[21] P. Lindqvist, On the equation $\operatorname{div}\left(\left|\nabla_{u}\right|^{p-2} \nabla_{u}\right)+|\lambda u|^{p-2} u=0$, Proc. Amer. Math. Soc. 109 (1990), 157-163.

[22] J. Appell, P. P. Zabrejko, Remarks on the superposition operator problem in various function spaces, Complex Var. Elliptic Equ. 55 (2010), 727-737.

[23] J. J. Ludew, On Nemytskij operator in the space of absolutely continuous set-valued functions, J. Appl. Anal. 17 (2011), 277-290.

[24] J. Matkowski, Functional equations and Nemytskii operators, Funkcialaj Ekvacioj. 25 (1982), 127-132.

[25] T. Runst, W. Sickel, Sobolev spaces of fractional order, Nemytskij Operators, and Nonlinear Partial Differential Equations, Berlin, New York, De Gruyter, 1996.

[26] G. Acosta, R. G. Duran, An optimal Poincare Inequality in $L^{1}$ for convex domains, Proc. Amer. Math. Soc. 132 (2020), 195-202.

[27] Y. Liu, The Poincaré inequality and quadratic transportation-variance inequalities, Electron. J. Probability 25 (2020), 1-16.

[28] J. Appell, E. De Pascale, A. Vignoli, Nonlinear spectral theory, Degruyter, Berlin, 2004. 